\title{
Efficacy of the inducing chemotherapy combined with synchronized chemotherapy and radiotherapy on the local advanced cervical cancer
}

\author{
Q. Xiong1* ${ }^{*}$ T. Zhang ${ }^{2}$, S. Su ${ }^{1}$ \\ ${ }^{1}$ Department of Gynecology, the Third Affiliated Hospital of Zhongshan University, Guangzhou, Guangdong510600, \\ China. \\ ${ }^{2}$ Department of Obstetrics and Gynecology, Guangzhou Women's and Children's Medical Center, Guangzhou, \\ Guangdong510623, China
}

- Original article

\author{
*Corresponding author: \\ Qian Xiong, Ph.D., \\ E-mail: \\ prof.qingnazhang@gmail.com
}

Revised: December 2020

Accepted: December 2020

Int. J. Radiat. Res., October 2021; 19(4): 1001-1007

DOI: 10.29242/ijrr.19.4.1001

\begin{abstract}
Background: To explore the efficacy of inducing chemotherapy in combination with the synchronized chemotherapy and radiotherapy on the local advanced cervical cancer. Materials and Methods: In this prospective cohort study, 212 patients with moderate or advanced cervical cancer who underwent their chemoradiotherapy were assessed. These patients were divided into the Group A, B and Group C. At 3 months, 1, 3, and 5 years after radiotherapy, efficacy and adverse effects evaluation were conducted. Data were analyzed using SPSS software. Results: Among Group A, B, and C, comparison over their short-term efficacy showed that the differences had no statistical significance $(P>0.05)$. The overall survival of patients in the Group A, B, and C was $90.12 \%, 85.29 \%$, and $91.75 \%$ in the $1^{\text {st }}$ year, $85.19 \%, 79.41 \%$, and $88.66 \%$ in the $3^{\text {rd }}$ year, and $82.72 \%, 73.53 \%$ and $84.54 \%$ in the $5^{\text {th }}$ year; differences among these OS showed no statistical significance $(P>0.05)$. While the dose modification factor (DMF) rate in Group $C$ was higher than those in Group A and B $(P<0.05)$, showing its radioprotective effect. The incidence of the blood responses at Grade 3 or 4 in Group A was significantly lower than those in Group B and $C(P<0.05)$. Conclusion: Our results showed a radioprotective effect of inducing chemotherapy in combination with the synchronized chemotherapy and radiotherapy, while the possibility of risk of blood responses was relatively high. So further studies assessing blood reactions are recommended in the long term.
\end{abstract}

Keywords: Cervical cancer, inducing chemotherapy, synchronized chemotherapy and radiotherapy, efficacy.

\section{INTRODUCTION}

Cervical cancer is the most common malignancies in the female reproductive system, ranking only second to breast cancer in the prevalence and first in all-female malignancies in the mortality rate. In recent years, younger women are more prone to cervical cancer which severely threatens the health and life quality (1). Besides, the majority of patients have progressed into the advanced stage, and have no choice but radiotherapy. Increasing evidence has shown that the synchronized chemotherapy and radiotherapy excel in the improvement of local control rate when comparing to the single radiotherapy (2), with a decrease in the risk of death but an increase in the survival rate of patients and improvement in prognosis (3).

At present, synchronized chemotherapy and radiotherapy are the standard patterns in the treatment of advanced local cervical cancer (4). Despite that synchronized chemotherapy and radiotherapy can ameliorate the prognosis of cervical cancer, cervical cancer-related death in 
the developing countries still ranks first in all malignancies, which may be correlated with the difficulty in eliminating the tumor stem cells and micrometastasis lesions. To further improve the prognosis of cervical cancer after synchronized chemotherapy and radiotherapy, researchers have put forward a variety of protocols, but there remains no standard protocol. Additionally, previous literature has noted that studies on the prognosis of cervical cancer were conducted on the single treatment and arrived at no unified conclusion over the efficacy and prognosis of different treatment patterns (5).

The literature review showed that Synchronized Chemotherapy may improve survival in women with cervical cancer and affect recurrence (6); while Complementary radiation therapy may be associated with an increased risk of acute toxicity (7). However, due to controversies among the studies and the lack of long-term data, it is not clear whether this could be modified by decreasing radiotherapy dosage following the Synchronized Chemotherapy (8).

As the evidence is limited by the small number of the previous studies and the optimal treatment strategy for advanced local cervical cancer is not yet demonstrated (8), in this study, we compared the efficacy of different treatment protocols (chemotherapy in combination with the synchronized chemotherapy and radiotherapy)on the local moderate or advanced cervical cancer to identify the best pattern and provide theoretical evidence for clinical treatment of moderate or advanced local squamous cancer of the cervix.

\section{MATERIALS AND METHODS}

In this prospective cohort study patients with moderate or advanced cervical cancer who underwent their chemoradiotherapy were assessed. This study was based on ethics in the research committee of the Third Affiliated Hospital of Zhongshan University and was based on the Declaration of Helsinki. All participants fulfilled the informed consent.
Inclusion criteria in this study were patients receiving initial treatment for Stage IIB to IVb of squamous cancer of the cervix; with no confirmed other tumors nor lymphatic metastasis through MRI. Also, only patients having tumor diameter $>4 \mathrm{~cm}$ were included.

Exclusion criteria were as follows: being diagnosed by other types of tumors; need to receive pelvic radiotherapy or chemotherapy and lymph nodes getting involved.

According to the criteria above, we selected a total of 212 patients with moderate or advanced cervical cancer who underwent their initial treatment in this hospital between Oct 2016 and Sep 2018. All subjects aged between 35 and 70 years old, with an average of 55.8 years old, and a median of 49 years old. The scores of the performance status (PS) were all not lower than 7 points, and there were 138 patients in Stage IIB, 10 in Stage IIIA, 59 in Stage IIIB, and 5 in Stage IV according to the clinical staging criteria of the International Federation Obstetrics (FIGO) in 2009.

All of the subjects had a complete pathological diagnosis, clinical data, and follow-up data, and took the radical radiotherapy for the first time. Before the radiotherapy, they were all diagnosed with the squamous carcinoma of the cervix, in which 63 patients had poorly differentiated tumors, 112 had a moderately differentiated tumor and 37 had a highly differentiated tumor. The diameter of the tumor was longer than $4 \mathrm{~cm}$, and according to the treatment pattern, based on their treatment protocol which was implicated by the specialist, they were divided into the Group A (control, synchronized chemotherapy, and radiotherapy, $\mathrm{n}=82$ ), Group $\mathrm{B}$ (contro), synchronized chemotherapy, and radiotherapy in combination with adjuvant chemotherapy, $\mathrm{n}=32$ ) and Group C (observation, inducing chemotherapy in combination with the synchronized chemotherapy and radiotherapy, $\mathrm{n}=98$ ).

Comparisons of the general data and pathological stages of patients among the three groups showed that the differences had statistical significance $(P>0.05$; table 1$)$. 
Table 1. General data of the patients in Group A, B, and C.

\begin{tabular}{|c|c|c|c|}
\hline & $\begin{array}{c}\text { Group A } \\
(\mathbf{n = 8 1})\end{array}$ & $\begin{array}{c}\text { Group B } \\
(\mathbf{n = 3 4 )}\end{array}$ & $\begin{array}{c}\text { Group C } \\
\text { (n=97) }\end{array}$ \\
\hline Age (years) & $\begin{array}{c}36-69 \\
(55.6)\end{array}$ & $\begin{array}{c}35-69 \\
(55.9)\end{array}$ & $\begin{array}{c}36-70 \\
(60.0)\end{array}$ \\
\hline $\begin{array}{c}\text { Pathological } \\
\text { stages (n): }\end{array}$ & 23 & 11 & 29 \\
\hline $\begin{array}{c}\text { Low } \\
\text { differentiation }\end{array}$ & 24 & 18 & 50 \\
\hline $\begin{array}{c}\text { Moderate } \\
\text { differentiation }\end{array}$ & 44 & 5 & 18 \\
\hline $\begin{array}{c}\text { High } \\
\text { differentiation }\end{array}$ & 14 & & \\
\hline
\end{tabular}

\section{Radiation and chemotherapy methods}

Radiotherapy procedure was carried out through the extrapelvic radiation plus the intrapelvic brachytherapy (Elekta, brochure code: 888.00414 MKT), with the radical dosage and the medical accelerator. Initially, penetrating radiation was performed over the anterior and posterior pelvic fields using the $6 \mathrm{MVX}$ ray vertically from the upper edge of the $5^{\text {th }}$ lumber disk to the lower edge of the obturator, and horizontally at 1 to $2 \mathrm{~cm}$ from the external edges of the pelvis. Extrapelvic radiation was performed once per day, five times a week in a total dosage of DT46Gy/23 times, during which a central shelter lead (10 $\mathrm{cm} \times 4 \mathrm{~cm}$ ) was placed after the 12 times of radiation, and intrapelvic brachytherapy was conducted twice a week after 15 times of extrapelvic radiation, 6Gy/time.

For patients in Group A, they received the intravenous infusion of cis-platinum (Qilu Pharmaceutical Co., Ltd; Batch No.: 1A1A1412030B) at $40 \mathrm{mg} / \mathrm{m}^{2}$, once per week and lasting for six weeks, or cis-platinum in combination with the paclitaxel (Yangtze River Pharmaceutical Group; Batch No.: 14111911) for 2 cycles in synchronization (paclitaxel: 135-175 $\mathrm{mg} / \mathrm{m}^{2}$, intravenous infusion; D1; cis-platinum: $60-80 \mathrm{mg} / \mathrm{m}^{2}$, intravenous infusion, D2: 21 days for 1 cycle, and replicate from Day 21 to 28); for patients in Group B, in addition to the protocols of Group A, they additionally took 1 or 2 cycles of TP protocol for chemotherapy, with the dose coinciding with the inducing chemotherapy; in Group C, patients underwent the TP protocol for inducing chemotherapy (paclitaxel: $135-175 \mathrm{mg} / \mathrm{m}^{2}$, intravenous infusion; D1; cis-platinum: $60-80 \mathrm{mg} / \mathrm{m}^{2}$, intravenous infusion, D2: 21 days for 1 cycle, and replicate from Day 21 to 28) prior to the radiotherapy for 1 or 2 cycles, and thereafter, they took the protocols same as the Group A.

\section{Evaluation of efficacy}

A comprehensive evaluation was performed before, at 3 months, 1,3 , and 5 years after radiotherapy. (1) Short-term efficacy: Patients were required to visit the clinic at 3 months after treatment for evaluation of the efficacy complete remission: no tumor; partial remission: tumor shrinkage more than 50\%; stable disease: tumor enlargement or shrinkage, not more than 25\%; progression disease: tumor enlargement over $25 \%$ or findings of new lesions. Patients with CR or PR were considered effective, while those with PD or SD were considered non-effective. (2) Long-term efficacy: Evaluation was carried out with the overall survival rates, PFS rates, local control rates, and DMF rates at the $1^{\text {st }}, 3^{\text {rd }}$, and $5^{\text {th }}$ year after treatment.

\section{Follow-up}

Follow-up duration lasted from the beginning of the chemotherapy or radiotherapy at admission to the death of patients or the end of follow-up (June $31^{\text {st }}, 2018$ ). Follow-up was mainly carried out through reviewing the clinic data, telephone, or correspondences, or if the methods failed, we corresponded to the working place or the police station according to the address. Any patients without the follow-up information were deemed as the loss to the follow-up. As a result, follow-up duration ranged from 10 to 89 months, with a median of 49 months. All patients underwent the follow-up.

\section{Evaluation of the toxic effect}

Adverse reactions in chemotherapy were assessed according to the CTCAE3.0 criteria issued by $\mathrm{NCI}{ }^{(9)}$. The acute responses to the radiotherapy according to the Grading Criteria of the Acute Radiation Injury of RTOG (9). The radiation injury in the advanced stage was evaluated according to the Grading Criteria of 
the Radiation Injury of RTOG/EORTC (9). Differences in the evaluation were compared among the three groups.

\section{Statistical methods}

Statistical analysis was performed by using the SPSS 22.0 software. Enumeration data were displayed as a percentage (\%). For data with differences of statistical significance detected by the Kruskal Walls test, data were compared by the pairwise comparison by using the chi-square test. $\alpha=0.05$ was set as the inspection level.

\section{RESULTS}

\section{Comparison of the short-term efficacy}

Follow-up at a clinic or telephone terminated on June $31^{\text {st }}, 2018$, with a follow-up rate of $100 \%$. The effective rates of Group A, B, and C were $93.90 \%, 96.88 \%$, and $94.90 \%$, without any statistically significant difference $\left(\chi^{2}=0.414, \mathrm{P}<\right.$ $0.813)$.

\section{Comparisons of the PFS rate, local control rate, and the DMF rate}

As shown in table 2, no statistical significance

Table 2. Comparisons of the PFS rate, local control rate and the DMF rate $[\mathrm{n}(\%)]$.

\begin{tabular}{|c|c|c|c|c|}
\hline Group & $\mathrm{n}$ & PFS rate & Local control rate & DMF rate \\
\hline Group A & 81 & $55(67.90)$ & $70(86.42)$ & $57(70.37)$ \\
\hline Group B & 34 & $23(67.65)$ & $29(85.29)$ & $24(70.59)^{\mathrm{ab}}$ \\
\hline Group C & 97 & $73(75.26)$ & $85(87.63)$ & $93(95.88)^{\mathrm{c}}$ \\
\hline $\mathrm{c}^{2}$ & & 1.253 & 0.016 & 20.291 \\
\hline$P$ & & 0.535 & 0.992 & 0.001 \\
\hline
\end{tabular}

Note: a $P<0.001$ vs. Group C; $b$ P $=0.705$ vs. Group A; c P $<0.001$ vs. Group A. was identified in the differences of PFS rates and local control rate among three groups $(\mathrm{P}>0.05)$, but as for the DMF rates, the difference had statistical significance $(P<0.05)$, among which the DMF rate in Group $\mathrm{C}$ was higher than those in the Group A and B $(\mathrm{P}<0.05)$.

\section{Comparison of the survival}

As shown in table 3 , no statistical significance was found in the differences in 1-, 3- and 5-year survival rates of patients among the three groups $(\mathrm{P}>0.05)$.

\section{Comparison of the incidence of adverse reactions}

In table 4 , the incidence rate of the blood responses at Grade 3 or 4 in Group A was significantly lower than those in Group B and C $(\mathrm{P}<0.05)$. No gastrointestinal responses at Grade 3 or 4, or liver injury at Grade 3 or 4 was identified in all subjects, and the differences of the incidences of the blood responses, gastrointestinal responses, or liver injury at Grade 1 or 2 showed no statistical significance $(\mathrm{P}>0.05)$.

Table 3. Comparison of the survival rates of patients among three groups [n (\%)].

\begin{tabular}{|c|c|c|c|c|}
\hline Group & $\mathrm{n}$ & 1-year survival & 3-year survival & 5-year survival \\
\hline Group A & 81 & $73(90.12)$ & $69(85.19)$ & $67(82.72)$ \\
\hline Group B & 34 & $29(85.29)$ & $27(79.41)$ & $25(73.53)^{\mathrm{a}}$ \\
\hline Group C & 97 & $89(91.75)$ & $86(88.66)$ & $82(84.54)^{\mathrm{b}}$ \\
\hline $\mathrm{c}^{2}$ & & 0.694 & 0.611 & 1.292 \\
\hline$P$ & & 0.707 & 0.737 & 0.525 \\
\hline
\end{tabular}

Note: a P $<0.005$ vs. Group C; $b$ P $<0.005$ vs. Group A.

Table 4. Comparison of the incidences of the adverse responses of patients among three groups [n (\%)].

\begin{tabular}{|c|l|c|c|c|c|c|}
\hline \multicolumn{2}{|c|}{ Adverse responses } & Group A(n=81) & Group B $(\mathrm{n}=34)$ & Group C $(\mathrm{n}=97)$ & $\chi^{2}$ & $P$ \\
\hline \multirow{2}{*}{ Blood responses } & Grade 1 or 2 & $48(59.26)$ & $24(70.59)$ & $72(74.23)$ & 4.103 & $>0.05$ \\
\cline { 2 - 7 } & Grade 3 or 4 & $6(7.41)$ & $9(26.47)^{\text {ab }}$ & $19(19.59)^{\mathrm{c}}$ & 6.122 & $<0.05$ \\
\hline \multirow{2}{*}{ Gastrointestinal responses } & Grade 1 or 2 & $9(11.11)$ & $5(14.71)$ & $15(15.46)$ & 0.421 & $>0.05$ \\
\cline { 2 - 7 } & Grade 3 or 4 & 0 & 0 & 0 & - & - \\
\hline \multirow{2}{*}{ Liver injury } & Grade 1 or 2 & $9(11.11)$ & $4(11.76)$ & $13(13.40)$ & 0.217 & $>0.05$ \\
\cline { 2 - 7 } & Grade 3 or 4 & 0 & 0 & 0 & - & - \\
\hline
\end{tabular}

Note: $\mathrm{aP}=0.498$ vs. Group $\mathrm{C}\left(\chi^{2}=0.461\right) ; \mathrm{bP}=0.020$ vs. Group $\mathrm{A}\left(\chi^{2}=5.459\right) ; \mathrm{cP}=0.040$ vs. Group $\mathrm{A}\left(\chi^{2}=4.253\right)$. 


\section{DISCUSSION}

Increasing evidence has proved the efficacy of synchronized chemotherapy and radiotherapy on improving the survival rate and reducing the recurrence of moderate or advanced cervical cancer (10), and this strategy has already been frequently applied in the treatment of moderate or advanced cervical cancer (11). It has been reported that neoadjuvant chemotherapy can suppress tumor growth through down-regulating the Ki-67 and increasing the apoptosis, with decreases in the activity of tumor cells (11). According to the study of Wang D et al., they found that neoadjuvant chemotherapy can increase the 2-year survival rate of the advanced local cervical cancer patients to $93 \%$, while among those with poor prognosis, the 22-months survival rate to $81 \%$. Adverse responses are main characterized by blood toxicity, and neoadjuvant chemotherapy can decrease the risk of disease progression and increase the survival rate. It has also been shown that neoadjuvant chemotherapy can mitigate the distant metastasis of cervical cancer patients while increasing the 3-year survival rate $(12,13)$. Katsumata et al. (14) also noted that women aged below 35 years old are more sensitive to the elder women, with a significant increase in the 5-year disease-free survival rate and overall survival rate.

Recently, to reduce the distant metastasis and local recurrence of the advanced local cervical cancer after chemotherapy or radiotherapy, many scholars suggested continuing the chemotherapy for 2 or 3 cycles after synchronized chemotherapy and radiotherapy (15), with significant divergence in outcomes. In a study of 102 advanced local cervical cancer patients, in patients who underwent adjuvant chemotherapy after the synchronized chemotherapy and radiotherapy, or only the latter, the 2-year survival rates were $96.2 \%$ and $82 \%$, and the 3 -year survival rates were $86.5 \%$ and $70.0 \%$; and the local recurrence rate and distant metastasis rate in the former were significantly lower than those in the latter; moreover, comparisons of the short-term effective rates, 1-year survival rates and incidence rates of adverse responses showed that the differences had no statistical significance; thus, consolidation chemotherapy after synchronized chemotherapy and radiotherapy for the moderate or advanced cervical cancer is expected to improve the prognosis and increase the overall survival rate (16). Also, some scholars have found that the toxicity of chemotherapy would be amplified in the consolidation of chemotherapy after synchronized chemotherapy and radiotherapy, without any improvement in the PFS or OS (17). Eskander et al. (18) also believed that consolidation chemotherapy after synchronized chemotherapy and radiotherapy may exacerbate the toxicity to the blood system in addition to the uncertain efficacy of neoadjuvant chemotherapy on the advanced local cervical cancer.

In this study, we compared the efficacy and toxicity of different treatment methods among three groups and found that inducing chemotherapy plus the synchronized chemotherapy and radiotherapy can prolong the PFS, and excelled in the DMF among three groups, with tolerable adverse responses, similar to the previous pieces of literature $(19,20)$. Synchronized chemotherapy and radiotherapy followed by the neoadjuvant chemotherapy did not manifest any additional advantages, which may correlate with other factors: (1) Before the radiotherapy, the abundant vessels of the tumor may precisely deliver the chemotherapeutics to the tumor cells; (2) Inducing chemotherapy, due to the cytotoxic effect, performs well in reducing the tumor volume and decreases the proportion of hypoxic cells that were not sensitive to the radiotherapy; (3) Inducing chemotherapy could suppress the proliferation of tumor cells, while facilitating the apoptosis, thereby decreasing the tumor stem cells; (4) After the synchronized chemotherapy and radiotherapy, the occluded vessels in the pelvic tissues due to the fibrosis limited the diffusion and distribution of chemotherapeutics in the adjuvant chemotherapy.

\section{Limitations of the study}

Thus, through this study, we believed that one or two cycles of inducing chemotherapy 
before the synchronized chemotherapy and radiotherapy may benefit the patients with the advanced local squamous carcinoma of the cervix, but it remains unknown whether the adjuvant chemotherapy after the synchronized chemotherapy and radiotherapy can further benefit the patients since the conclusion should be validated by expanding the sample size.

\section{CONCLUSION}

For the moderate or advanced squamous carcinoma of the cervix, inducing chemotherapy in combination with the synchronized chemotherapy and radiotherapy may increase the distant-metastasis-free rate, and prolong the overall survival rate. Thus, it is an ideal choice for the clinical treatment of advanced local cervical cancer. However, more effort is required to develop the best way to integrate chemotherapy into the precise radiotherapy to further increase the survival rate and improve the life quality of patients.

\section{Conflicts of interest: Declared none.}

\section{REFERENCES}

1. Prakash G and Kumar L (2011) Chemotherapy for cervical cancer: an update; in Rajaram S (ed) Cancer Cervix, ed 1. New Dehli, Jaypee Brothers Medical Publishers Pvt. Ltd., pp 215-221.

2. Monk BJ, Sill MW, McMeekin DS, Cohn DE, Ramondetta LM, Boardman CH, Benda J, Cella D (2009) Phase III trial of four cisplatin-containing doublet combinations in stage IVB, recurrent, or persistent cervical carcinoma: a Gynecologic Oncology Group study. J Clin Oncol, 27: 4649-4655.

3. Kitagawa R, Katsumata N, Shibata T, Kamura T, Kasamatsu T, Nakanishi T, et al. (2015) Paclitaxel plus carboplatin versus paclitaxel plus cisplatin (TP) in metastatic or recurrent cervical cancer. The openlabel randomized phase III trial (JCOG0505). J Clin Oncol, 33: 2129-2135.

4. Scatchard K, Forrest JL, Flubacher M, Cornes P, Williams C (2012) Chemotherapy for metastatic and recurrent cervical cancer. Cochrane Database Syst
Rev, 10: CD006469.

5. Kumar L, Pramanik R, Kumar S, Bhatla N, Malik S (2015) Neoadjuvant chemotherapy in gynecological cancers - implications for staging. Best Pract Res Clin Obstet Gynaecol, 29: 790-801.

6. Gupta S, Maheshwari A, Parab P, Mahantshetty U, Hawaldar R, Sastri S, et al. (2018) Neoadjuvant chemotherapy followed by radical surgery versus concomitant chemotherapy and radiotherapy in patients with stage IB2, IIA, or IIB squamous cervical cancer: a randomized controlled trial. Journal of Clinical Oncology, 36(16): 1548-55.

7. Kumar L, Harish P, Malik PS, Khurana S (2018) Chemotherapy and targeted therapy in the management of cervical cancer. Current Problems in Cancer, 42 (2): 120-8.

8. Marchetti C, Fagotti A, Tombolini V, Scambia G, De Felice $F(2020)$ Survival and toxicity in neoadjuvant chemotherapy plus surgery versus definitive chemoradiotherapy for cervical cancer: A systematic review and meta-analysis. Cancer Treatment Reviews, 83: 101945.

9. Katsumata N, Yoshikawa $H$, Kobayashi $H$, Saito $T$, Kuzuya K, Nakanishi T, et al. (2013) Phase III randomized controlled trial of neoadjuvant chemotherapy plus radical surgery vs radical surgery alone for stages IB2, IIA2, and IIB cervical cancer: a Japan Clinical Oncology Group trial (JCOG 0102). Brit J Cancer, 108: 1957-1963.

10. Kim HS, Sardi JE, Katsumata N, Ryu HS, Nam JH, Chung $\mathrm{HH}$, et al. (2013) Efficacy of neoadjuvant chemotherapy in patients with FIGO stage IB1 to IIA cervical cancer: an International collaborative metaanalysis. Eur J Surg Oncol, 39: 115-124.

11. Rydzewska L, Tierney J, Vale CL, Symonds PR (2012) Neoadjuvant chemotherapy plus surgery versus surgery for cervical cancer. Cochrane Database Syst Rev, 12: CD007406.

12. Wang D, Yang J, Shen K, Xiang Y (2013) Neoadjuvant chemotherapy followed by fertility sparing surgery for women with stage IB1 cervical cancer. J Gynecol Oncol, 24: 287-290.

13. Petrelli F, De Stefani A, Raspagliesi F, Lorusso D, Barni S (2014) Radiotherapy with concurrent cisplatin based doublet or weekly cisplatin for cervical cancer: a systemic review and meta-analysis. Gynecol Oncol, 134: 166-171.

14. Ready NE, Rathore R, Johnson TT, Nadeem A, Chougule P, Ruhl C, et al. (2012) Weekly paclitaxel and carboplatin induction chemotherapy followed by concurrent chemoradiotherapy in locally advanced squamous cell carcinoma of the head and

Int. J. Radiat. Res., Vol. 19 No. 4, October 2021 
neck. Am J Clin Oncol, 35: 6-12.

15. Katsumata N, Yasuda M, Takahashi F, Isonishi S, Jobo T, Aoki D, et al. (2009) Dose-dense paclitaxel once a week in combination with carboplatin every 3 weeks for advanced ovarian cancer: a phase 3, open-label, randomized controlled trial. Lancet, 374: 1331-1338.

16. Singh RB, Chander S, Mohanti BK, Pathy S, Kumar S, Bhatla N, et al. (2013) Neoadjuvant chemotherapy with weekly paclitaxel and carboplatin followed by chemoradiation in locally advanced cervical carcinoma: a pilot study. Gynecol Oncol, 129: 124-128.

17. McCormack M, Kadalayil L, Hackshaw A, Hall-Craggs MA, Symonds RP, Warwick V, et al. (2013) A phase II study of weekly neoadjuvant chemotherapy fol- lowed by radical chemoradiation for locally advanced cervical cancer. Br J Cancer, 108: 2464-2469.

18. Eskander RN and Tewari KS (2014) Beyond angiogenesis blockade: targeted therapy for advanced cervical cancer. J Gynecol Oncol, 25: 249-259.

19.Zagouri F, Sergentanis TN, Chrysikos D, Filipits M, Bartsch R (2012) Molecularly targeted therapies in cervical cancer. A systematic review. Gynecol Oncol, 126: 291-303.

20. Falcetta FS, Medeiros LR, Edelweiss MI, Pohlmann PR, Stein AT, Rosa DD (2012) Adjuvant platinumbased chemotherapy for early stage cervical cancer. Cochrane Database Syst Rev, 6: CD005342. 
